picture. Azacitidine has been dropped from clinical use, but a preliminary report of treatment with hydroxyurea is promising. In three patients there was a $2 \cdot 3$-fold to 16 -fold increase in the percentage of haemoglobin $F$ and a virtual cessation of painful crises. ${ }^{9}$ The results of the careful controlled study of hydroxyurea being undertaken for the National Institutes of Health are awaited with interest, although the way hydroxyurea raises the haemoglobin $\mathrm{F}$ concentration is still incompletely understood.

Bone marrow transplantation for sickle cell disease has now been performed in a few centres, mainly in Belgium. ${ }^{10}$ Though transplantation is curative, it raises ethical issues that have yet to be fully discussed by either health professionals or patients in Britain. We need to explore these, as for $\beta$ thalassaemia major.

There is an urgent need in Britain to develop a structure for developing coordinated trials of new treatments for sickle cell disease. As a model haematologists have the excellently run trials for the leukaemias sponsored by the Medical Research Council. These not only produced valuable scientific infor- mation but also enabled physicians with only a few patients under their care to use up to date treatments.

Consultant Haematologist,

Central Middlesex Hospital,

London NW10 7NS

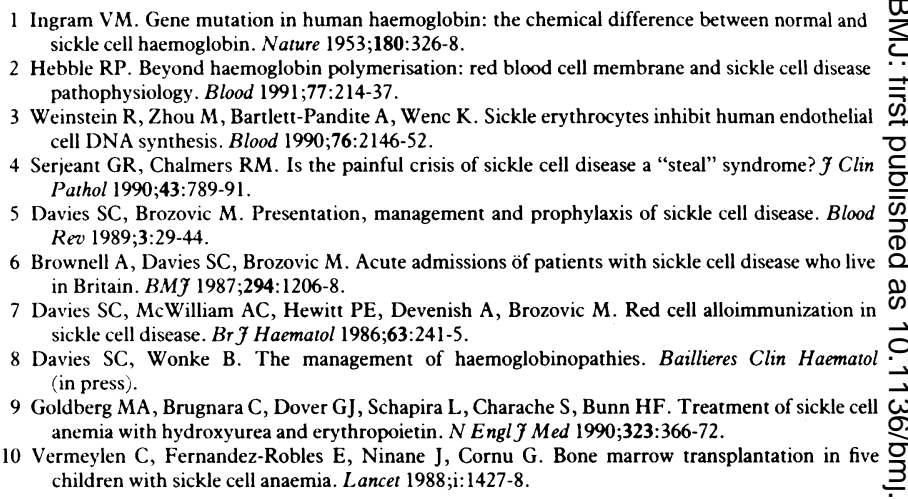

\title{
Hepatitis A
}

\section{Changing prevalence and possible vaccines}

Although hepatitis A is generally a benign infection, it may have a protracted or relapsing course, ${ }^{12}$ and, very rarely, result in fulminant hepatic failure. Its severity increases with age; in England and Wales in 1980-8 the fatality rate was 0.02 $0.03 \%$ in those aged under 55 but $1.5 \%$ in those aged over $64 .^{3}$

Hepatitis A virus is exceptionally stable and is spread by the faecal-oral route, either from person to person or through contaminated food or water. In countries with poor sanitation this is common-with almost universal infection of young children resulting in little clinical disease and lifelong immunity. As standards of hygiene improve, however, infection is delayed, and many escape early asymptomatic infection only to develop clinical hepatitis A as adolescents or adults. This emergence of the disease at an older age is one of several factors giving a sharper edge to the search for a vaccine.

In many developed countries the prevalence of immunity is related to age and is falling. Among medical students in London the prevalence of hepatitis A IgG fell from $14 \%$ in $1980^{4}$ to $6 \%$ in 1990 (unpublished findings), and in blood donors in London it fell from $47 \%$ in 1977 to $32 \%$ in 1985.5 Among 863 Foreign Office staff the overall prevalence of hepatitis A IgG in $1989-90$ was $39 \%$, being $43 \%$ in those over 40 but only $14 \%$ in younger people (unpublished findings).

The incidence of hepatitis $\mathrm{A}$ is difficult if not impossible to measure, as many cases are asymptomatic and there is considerable underreporting. Despite the decreasing seroprevalence, notifications and laboratory reports of hepatitis A to the Public Health Laboratory Service Communicable Disease Surveillance Centre increased between 1987 and 1990. Although this may reflect increased reporting, it is more likely that infection is spreading in the community. Indeed, hepatitis A virus infection is now endemic in several deprived urban areas including Liverpool - where it has been endemic in children for over a year ( $Q$ Syed, personal communication). If this trend continues hepatitis A may become a marker of inner city deprivation.

Hepatitis A has traditionally been controlled by sewage disposal and better hygiene, while human normal immuno- $\vec{\emptyset}$ globulin has been used in developed countries as prophylaxis. both before and after exposure. In the United Kingdom use of ${ }_{0}$ this preparation has increased recently, mostly because of travel to endemic countries. About 1.5 million people visit $\overline{0}$ such areas annually, about 600000 for the first time ( $M \stackrel{0}{\circ}$ Capaldi, personal communication); in 1990 the number of doses of human normal immunoglobulin issued approached $\overrightarrow{\overrightarrow{0}}$ 600000 . This plasma product is safe and effective, but it may become less effective with the declining prevalence of immunity to hepatitis A. Furthermore, it is costly and gives only shortlived protection.

A vaccine would have obvious advantages. Several types are possible, but conventional inactivated vaccines and live 3 attenuated vaccines have received most attention. Initial studies in primates with live attenuated vaccines identified several variants that were safe, immunogenic, and protec-o tive.$^{67}$ In human volunteers some difficulty was experienced in achieving a balance between immunogenicity and attenuation, ${ }^{8-10}$ but encouraging results have been reported by $a$ 을 Chinese group using a different attenuated strain, which induced high seroconversion rates without serious side N effects. ${ }^{1112}$ Furthermore, this vaccine provided protection ${ }_{\sigma}^{\omega}$ under natural conditions. In all these studies live vaccine has been given by injection; the oral route would be preferable but $\frac{}{\mathbb{D}}$ so far results have been disappointing. Points of concern $\stackrel{?}{+}$ include excretion and transmission of the virus, reversion to 0 virulence, and the possibility of persistence in the liver or $\frac{\vec{\Phi}}{\vec{D}}$ elsewhere. Moreover, a chemical or genetic marker for $\frac{\widehat{\Phi}}{\Phi}$ attenuation remains to be identified.

Studies with inactivated vaccines in large numbers of human volunteers have shown that these vaccines are safe, 20 induce high seroconversion rates with just two doses givens one month apart, and induce neutralising antibodies at $\frac{\bar{\partial}}{\partial}$ concentrations that usually exceed the peak concentration. after one dose of human normal immunoglobulin. ${ }^{13-15} \mathrm{~A}$ third dose results in higher concentrations of antibody, which should provide long term protection. As with hepatitis B 
vaccine, older volunteers and men tend to respond less well (unpublished findings). The important question of whether the vaccine is protective remains to be answered as studies are difficult to implement. Nevertheless, as human normal immunoglobulin is effective a vaccine that produces neutralising antibodies should also protect against disease.

Where and how will these vaccines be used? The World Health Organisation has incorporated hepatitis A into its programme for future vaccine development, since this vaccine would be of benefit to countries experiencing improved standards of hygiene and an increase in symptomatic hepatitis $A$ in young adults. In Shanghai in 1988, for example, there were 310746 reported cases of hepatitis A, $84 \%$ occurring in those aged 20-29."

In developed countries a vaccine would obviate the use of human normal immunoglobulin in, for example, members of the armed forces and travellers to endemic areas. Other high risk groups include people working with children, male homosexuals, and intravenous drug abusers. ${ }^{16}$ Food handlers may also be a group to be targeted as they are often the source of outbreaks. Whether an inactivated vaccine would reduce virus excretion, however, remains to be established.

Initially, the cost of a three dose course of inactivated hepatitis A vaccine is likely to be similar to that of hepatitis $B$ vaccine, reflecting high production costs and a poor yield of virus in cell cultures. In developing countries this would be unacceptably high, and techniques that produce a higher virus yield need to be developed. Alternatively, a live vaccine, which is likely to be cheaper, may be preferable in this setting. In developed countries, on the other hand, the inactivated vaccines currently on trial, despite their likely cost, would undoubtedly be used in preference to human normal immunoglobulin.

A J TILZEY

Senior Registrar in Virology JE BANATVALA

Professor of Clinical Virology

United Medical and Dental Schools of Guy's and St Thomas's Hospitals, St Thomas's Campus,

London SE1 7EH

1 Gruer LD, McKendrick MW, Beeching NJ, Geddes AM. Relapsing hepatitis associated with hepatitis A virus. Lancet 1982;ii: 163 .

2 Jacobson IM, Nath BJ, Dienstag JL. Relapsing viral hepatitis type A. 7 Med Virol 1985;16:163-9.

3 Clinical hepatitis A: laboratory reports years 1980-88. Communicable Disease Report 1990:89/46.

4 Banatvala JE, Thorogood RJ. Hepatitis A antibodies in London blood donors, medical students, and patients. Lancet 1980; ; 595.

5 Vandervelde EM, Millard JM, Parry JV, Mortimer PP. Time for action on hepatitis B

immunisation. BMF 1987;294:301.
6 Provost PV, Buynak EB, McLean AA, Hilleman MR, Scolnick EM. Progress toward a live $\overrightarrow{\overrightarrow{0}}$ attenuated human hepatitis A vaccine. In: Vyas GN, Fyrndysh JI, Hoffnagle JH, eds. Viral hepatitis and liver disease. Orlando: Grune and Stratton, 1984:467-75.

7 Provost PJ, Emini EA, Lewis JA, Gerety RJ. Progress toward the development of a hepatitis A vaccine. In: Zuckerman AJ, ed. Viral hepatitis and liver disease. New York: A R Riss Inc, 1988:83- O 6.

8 Ellis RW, Provost PJ. Hepatitis B and A vaccines. In: Zuckerman AJ, ed. Recent developments in prophylactic immunization. Dordrecht: Kluwer, 1989:181-209.

9 Flehmig B, Heinricy U, Pfisterer M. Prospects for a hepatitis A virus vaccine. Prog Med Virol N 1990;37:56-71.

10 Gust ID. Design of hepatitis A vaccines. Br Med Bull 1990;46:319-28

1 Mao JS, Dong DX, Zhang HY, Chon NL, Zhang MY, Huang HY, et al. Primary study of N attenuated live hepatitis A vaccine (H2 strain) in humans. F Infect Dis 1989;159:621-4.

12 Mao J. Development of live, attenuated hepatitis A vaccine (H2-strain). Vaccine 1990;8:523-4.

3 Flehmig B, Heinricy U, Pfisterer M. Immunogenicity of a killed hepatitis A vaccine in seronegative volunteers. Lancet 1989;i:1039-41.
Andre FE, Hepburn A, Hondt ED. Inactivated candidate vaccines for hepatitis A. Prog Med Virol 음 1990;37:72-95.

15 Wiedermann G, Ambrosch F, Kollaritsch H, Hofmann H, Kunz Ch, Hondt ED, et al. Safety and 6 immunogenicity of an inactivated hepatitis $\mathrm{A}$ candidate vaccine in healthy adult volunteers. Vaccine 1990:8:581-4.

16 Hepatitis A among drug abusers. MMWR 1988;37:297-301.

\section{Non-operative management of blunt splenic injury}

\section{Works well in about a quarter of patients}

Prompt splenectomy has traditionally been the treatment of choice for a spleen damaged by blunt abdominal trauma, but the wisdom of such an approach is being increasingly questioned. The risks and range of sepsis after splenectomy are now well defined, ' and a variety of options are available for preserving splenic function, including splenorraphy and partial splenectomy. ${ }^{2}$ There is also increasing evidence that some patients may not need surgery at all.

Non-operative management of blunt splenic injury in children is now a well accepted form of treatment in selected cases. In children particularly bleeding from the injured spleen has often stopped by the time laparotomy is performed. In 1968 paediatric surgeons in Toronto described 12 patients with suspected splenic injuries successfully managed without operation. ${ }^{3}$ By the mid-1980s about 260 children managed conservatively had been reported, with excellent results. ${ }^{4}$

Surgeons have been more reluctant to use selective nonoperative management in adults whose spleen has ruptured. This reluctance is undoubtedly partly due to the fact that sepsis after splenectomy is both less common and less dangerous in adults than in children. ${ }^{1}$ Also, changes in splenic architecture and vasculature with age ${ }^{56}$ and possible differences in the mechanisms of splenic injury between adults and children $^{7}$ may make the injured adult spleen less likely to stop bleeding spontaneously than a child's. These considerations, together with the possibility of missing associated injuries and fears of delayed rupture and the development of posttraumatic cysts or splenosis, still lead most surgeons to perform prompt splenectomy (or spleen preserving surgery) in adults.

Several recent reports, however, suggest that a sizeable proportion of adults with splenic injury after blunt trauma may be successfully managed without operation. Out of $45 \AA$ adults presenting with a ruptured spleen Klin et al selected 15 in a stable condition for conservative management, and none required operation. ${ }^{8}$ Villalba et al managed 34 out of 51 adult $N$ patients conservatively, only one requiring splenectomy, ${ }^{9}$ and $>$ Pachter et al managed 26 out of 146 conservatively, again with only one requiring surgery. ${ }^{10}$ Combining the results of these $\bar{N}$ three studies suggests that about one in four patients could be $\underset{\omega}{N}$ managed conservatively, with a success rate of over $95 \%$. 으 These results contrast with less encouraging reports from 0 smaller earlier studies, in which a non-operative approach was successful in only one in four selected patients. ${ }^{711}$

To achieve the best results selection criteria must be strict. Patients suitable for non-operative management should be $\vec{D}$ haemodynamically stable on admission or after initial resus- $\frac{\Omega}{\mathbb{Q}}$ citation. A ruptured spleen is usually suspected from clinical $\frac{\varrho}{\sigma}$ features suggestive of intra-abdominal bleeding and localised or generalised abdominal tenderness. The presence on plain $\delta$ radiography of fractured ribs overlying the spleen, an enlarged splenic shadow, and medial displacement of the gastric air bubble are also suggestive. Peritoneal lavage is often used to confirm the presence of intraperitoneal blood, and both ultrasound and laparoscopy are useful not only in confirming a splenic injury but also in helping to exclude other injuries. 